\title{
A new bright eclipsing hot subdwarf binary from the ASAS and SuperWASP surveys
}

\author{
V. Schaffenroth ${ }^{1,2}$, S. Geier ${ }^{1,3}$, H. Drechsel ${ }^{1}$, U. Heber ${ }^{1}$, P. Wils ${ }^{4}$, R. H. Østensen ${ }^{5}$, P. F. L. Maxted ${ }^{6}$, and G. di Scala ${ }^{7}$ \\ 1 Dr. Remeis-Observatory \& ECAP, Astronomical Institute, Friedrich-Alexander University Erlangen-Nürnberg, Sternwartstr. 7 , \\ 96049 Bamberg, Germany \\ e-mail: veronika.schaffenroth@sternwarte.uni-erlangen.de \\ 2 Institute for Astro- and Particle Physics, University of Innsbruck, Technikerstr. 25/8, 6020 Innsbruck, Austria \\ ${ }^{3}$ European Southern Observatory, Karl-Schwarzschild-Str. 2, 85748 Garching, Germany \\ 4 Vereniging voor Sterrenkunde, Belgium \\ 5 Institute of Astronomy, KU Leuven, Celestijnenlaan 200D, 3001 Heverlee, Belgium \\ 6 Astrophysics Group, Keele University, Staffordshire, ST5 5BG, UK \\ 7 Carnes Hill Observatory, 34 Perisher St., 2171 Horningsea Park, NSW, Sydney, Australia
}

Received 14 December 2012 / Accepted 1 March 2013

\begin{abstract}
We report the discovery of a bright $\left(m_{\mathrm{V}}=11.6 \mathrm{mag}\right)$, eclipsing, hot subdwarf binary of spectral type B with a late main sequence companion from the All Sky Automated Survey (ASAS 102322-3737.0). Such systems are called HW Vir stars after the prototype. The lightcurve shows a grazing eclipse and a strong reflection effect. An orbital period of $P=0.13927 \mathrm{~d}$, an inclination of $i=65.86^{\circ}$, a mass ratio $q=0.34$, a radial velocity semiamplitude $K_{1}=81.0 \mathrm{~km} \mathrm{~s}^{-1}$, and other parameters were derived from a combined spectroscopic and photometric analysis. The short period can only be explained by a common envelope origin of the system. The atmospheric parameters $\left(T_{\text {eff }}=28400 \mathrm{~K}, \log g=5.60\right)$ are consistent with a core helium-burning star located on the extreme horizontal branch. In agreement with that we derived the most likely sdB mass to be $M_{\mathrm{sdB}}=0.46 M_{\odot}$, close to the canonical mass of such objects. The companion is a late M-dwarf with a mass of $M_{\text {comp }}=0.16 M_{\odot}$. ASAS 102322-3737.0 is the third brightest of only 12 known HW Virginis systems, which makes it an ideal target for detailed spectroscopic studies and long-term photometric monitoring to search for period variations, e.g., those caused by a substellar companion.
\end{abstract}

Key words. subdwarfs - binaries: eclipsing - binaries: spectroscopic - stars: early-type - stars: fundamental parameters stars: individual: ASAS 102322-3737.0

\section{Introduction}

Hot subdwarf stars ( $\mathrm{sdB}$ ) are evolved, compact stars found in the disk and halo of our Galaxy. They dominate the population of faint blue stars. Especially in the context of Galaxy evolution, sdBs are important because they are believed to be the dominant source of the "UV upturn phenomenon" which is observed in elliptical galaxies (Brown et al. 1997, 2000). Subdwarf B stars are core helium-burning stars on the extreme horizontal branch (EHB). They have very thin hydrogen envelopes (Saffer et al. 1994; Heber 1986) that avoid hydrogen shell-burning. Therefore they evolve from the EHB directly to white dwarfs (Dorman et al. 1993).

The formation of sdBs requires an extraordinarily high mass loss on the red-giant branch (RGB). About half of the sdB stars are found in close binaries with periods from $\sim 0.05$ to $30 \mathrm{~d}$ (e.g. Maxted et al. 2001; Napiwotzki et al. 2004; Geier et al. 2012; Vennes et al. 2012). Mass transfer must therefore play an important role in the formation of these stars. In their binary evolution studies, Han et al. $(2002,2003)$ found that common-envelope evolution, resulting from dynamically unstable mass transfer near the tip of the RGB, should produce such short-period binaries $(P=0.1-10 \mathrm{~d})$. The most probable mass for such sdBs is $0.47 M_{\odot}$ (Han et al. 2003), which is called the canonical mass.
Eclipsing post-common envelope binaries that consist of sdBs and late $\mathrm{M}$ star companions with periods of about $2-3 \mathrm{~h}$ are called HW Vir systems after the prototype. Such systems are of high value because it is possible to derive the mass of the sdB, as well as the mass and the nature of the companion, from a combined analysis of time-resolved spectra and the lightcurve. These systems are rare but can easily be recognized by the prominent reflection effect, which is the only contribution of the companion to the optical light. Until now only twelve such systems have been known. Interest in HW Virginis systems has been revived by the discovery of substellar companions to the prototype HW Virginis (Lee et al. 2009) and HS 0705 (Qian et al. 2009) via the light-travel time method (for an up-to-date census see Zorotovic \& Schreiber 2013). For this method a long-term photometric monitoring for several years is necessary.

ASAS 102322-3737.0 (ASAS 10232 for short) was discovered as a variable star in the course of the All Sky Automated Survey by Pojmanski (2003), but misclassified as a $\delta$ Scuti star. In 2007 this system was found in the ASAS Survey by P. W. and recognized as an HW Virginis system. Another lightcurve was obtained at the Carnes Hill Observatory, Sydney, in March 2008. BVRI photometry was taken with the Euler telescope on La Silla in April 2008, confirming the presence of an eclipse. In the SuperWASP survey (Pollacco et al. 2006), this system was 
also observed in several runs. ASAS 10232 is the third brightest HW Vir system with $m_{\mathrm{V}}=11.6 \mathrm{mag}$, which makes it well suited for follow-up observations.

\section{Observations}

\subsection{Photometry}

Observations of ASAS 10232 were taken in the nights of the 10, 11, 12, 13, and 31 March 2008 in the $B, V$, and $I$ band with a 12" LX200 GPS Schmidt-Cassegrain Telescope at the Carnes Hill Observatory in Sydney, Australia. More lightcurves in BVRI were taken with the 1.2-m Leonhard Euler Telescope at the La Silla Obervatory, Chile, in the nights of the 23 and 25 April 2008. Moreover, a lightcurve of this system was observed with the robotic SuperWASP telescope at the South African Astronomical Observatory in three runs from May 2006 to January 2009. A broad band filter (400-800 nm) was used for this observation.

\subsection{Spectroscopy}

Fifteen medium-resolution spectra $(R=3400)$ of ASAS 10232 with exposure times of $70 \mathrm{~s}$ were obtained with the EMMI spectrograph at the ESO-NTT Telescope in La Silla, Chile, from the 11 to 14 January 2008 . These spectra cover only a small part of the optical spectrum (3900-4360 ̊) and are well suited to radial velocity work, but not to determining the atmospheric parameters. Another 33 spectra $(R \sim 1750,3800-6540 \AA)$ were thus obtained on the 20 and 25 February 2010 with the GMOS spectrograph mounted at the $8.1 \mathrm{~m}$ Gemini South telescope at Cerro Pachón Observatory in service mode with an exposure time between 100 and $300 \mathrm{~s}$. The EMMI data were reduced with the MIDAS package distributed by the European Southern Observatory (ESO). The GMOS data were reduced with the PAMELA $^{1}$ and MOLLY ${ }^{1}$ packages.

\section{Spectroscopic analysis}

The optical EMMI and GMOS spectra offer good phase coverage of the radial velocity curve. The GMOS spectra enabled us to determine the atmospheric parameters covering about half of the orbit.

\subsection{Radial velocity curve}

The radial velocities were measured by fitting a combination of Gaussians, Lorentzians, and polynomials to the Balmer and helium lines of all spectra. Assuming a circular orbit, sine curves were fitted to the RV data points in fine steps over a range of test periods. For each period, the $\chi^{2}$ of the best fitting sine curve was determined (see Geier et al. 2011a). At first the EMMIand the GMOS-datasets were fitted separately. In each case the best solution had the same orbital period as derived from the lightcurve $(P \simeq 0.139 \mathrm{~d})$. Both datasets cover either the full phase (EMMI) or about half of the phase (GMOS). While the RV-semiamplitudes are similar, the system velocities of the two datasets show a significant shift of about $27 \mathrm{~km} \mathrm{~s}^{-1}$ with respect to each other. This shift is probably an instrumental effect. More observations are needed to solve this issue.

\footnotetext{
1 http://www2 . warwick.ac.uk/fac/sci/physics/research/ astro/people/marsh/software
}

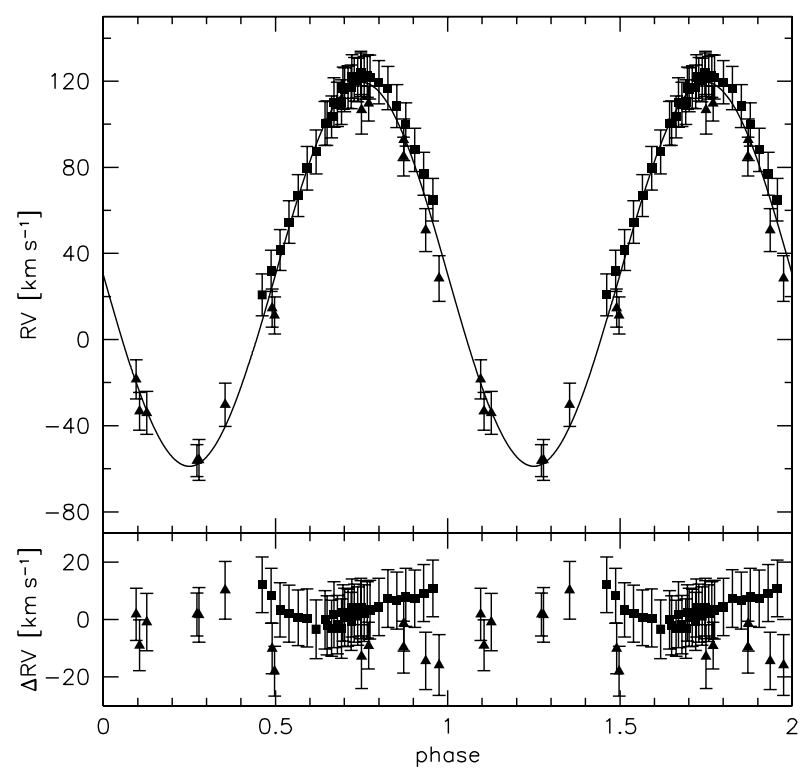

Fig. 1. Radial velocity plotted against orbital phase of ASAS 10232. The RV data was folded with the period measured from the SuperWASP lightcurve. The RVs were determined from ESO-NTT/EMMI spectra (triangle) and Gemini/GMOS spectra (rectangular). The errors are formal $1 \sigma$ uncertainties. The GMOS-RVs were shifted systematically to fit to the EMMI results.

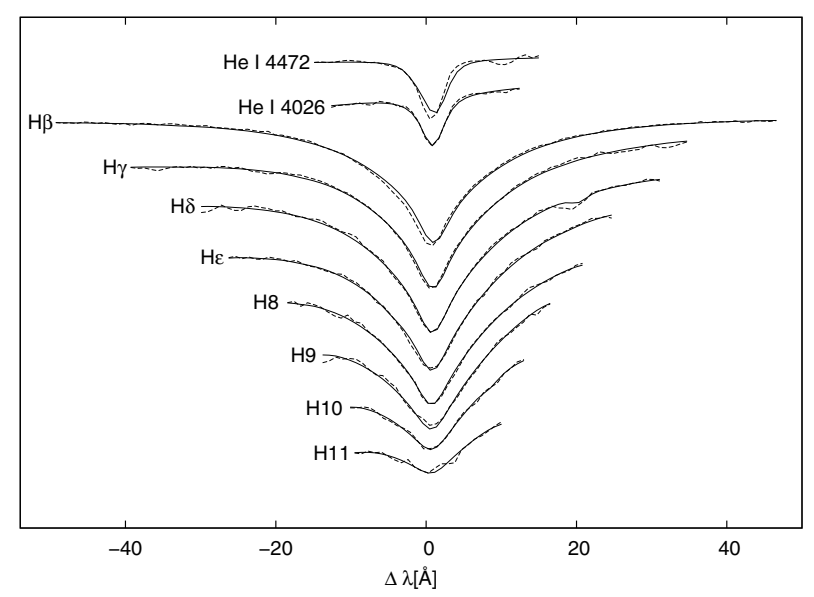

Fig. 2. Fit of the GMOS spectrum nearest to phase 0, the dashed line shows the measurement and the solid line shows the best fitting synthetic spectrum.

Taking the average value of the solutions derived from the two datasets, the semiamplitude of the radial velocity curve was determined to $K_{1}=81.0 \pm 3 \mathrm{~km} \mathrm{~s}^{-1}$. Figure 1 shows a phased $\mathrm{RV}$-curve where the radial velocities derived from the GMOS spectra have been shifted by $-27 \mathrm{~km} \mathrm{~s}^{-1}$.

\subsection{Atmospheric parameters}

Atmospheric parameters were determined by fitting synthetic spectra to the observed Balmer and helium lines of each of the 33 GMOS spectra using SPAS (Hirsch 2009). A model grid of synthetic spectra was calculated by using LTE model atmospheres with solar metalicity and metal line blanketting (Heber et al. 2000). Because some HW Vir systems showed an apparent change in the atmospheric parameters over the orbital phase 
V. Schaffenroth et al.: A new eclipsing hot subdwarf binary from the ASAS survey

Table 1. Radial velocities of ASAS 10232.

\begin{tabular}{|c|c|c|}
\hline Mid-HJD & $R V\left[\mathrm{~km} \mathrm{~s}^{-1}\right]$ & Instrument \\
\hline 2454476.71277 & $109.7 \pm 8.2$ & EMMI \\
\hline 2454476.72692 & $85.0 \pm 9.0$ & \\
\hline 2454476.79412 & $-30.3 \pm 10.0$ & \\
\hline 2454477.71635 & $28.4 \pm 10.6$ & \\
\hline 2454477.73450 & $-33.3 \pm 8.9$ & \\
\hline 2454477.75857 & $-55.8 \pm 9.5$ & \\
\hline 2454477.84140 & $92.8 \pm 9.7$ & \\
\hline 2454477.87249 & $-18.4 \pm 9.1$ & \\
\hline 2454477.87674 & $-34.0 \pm 10.0$ & \\
\hline 2454478.66009 & $106.6 \pm 11.2$ & \\
\hline 2454478.68602 & $50.9 \pm 10.0$ & \\
\hline 2454478.76423 & $11.2 \pm 8.7$ & \\
\hline 2454478.87209 & $-56.2 \pm 7.5$ & \\
\hline 2454479.73826 & $14.6 \pm 8.9$ & \\
\hline 2455247.69381 & $127.4 \pm 10.2$ & GMOS \\
\hline 2455247.69571 & $130.4 \pm 9.8$ & \\
\hline 2455247.69801 & $136.2 \pm 10.2$ & \\
\hline 2455247.69961 & $137.3 \pm 10.4$ & \\
\hline 2455247.70092 & $143.0 \pm 10.2$ & \\
\hline 2455247.70224 & $144.3 \pm 9.9$ & \\
\hline 2455247.70355 & $144.3 \pm 10.1$ & \\
\hline 2455247.70486 & $147.9 \pm 10.1$ & \\
\hline 2455247.70618 & $147.5 \pm 10.2$ & \\
\hline 2455247.70750 & $149.9 \pm 10.2$ & \\
\hline 2455247.70881 & $148.6 \pm 9.9$ & \\
\hline 2455247.71013 & $149.3 \pm 9.8$ & \\
\hline 2455252.68221 & $47.8 \pm 9.7$ & \\
\hline 2455252.68584 & $58.9 \pm 9.6$ & \\
\hline 2455252.68947 & $68.6 \pm 9.5$ & \\
\hline 2455252.69310 & $81.6 \pm 9.9$ & \\
\hline 2455252.69673 & $93.9 \pm 9.7$ & \\
\hline 2455252.70036 & $106.6 \pm 10.2$ & \\
\hline 2455252.70399 & $114.2 \pm 10.3$ & \\
\hline 2455252.70762 & $127.4 \pm 10.2$ & \\
\hline 2455252.71125 & $137.2 \pm 11.5$ & \\
\hline 2455252.71488 & $143.7 \pm 9.9$ & \\
\hline 2455252.71851 & $149.1 \pm 10.1$ & \\
\hline 2455252.72214 & $150.8 \pm 10.1$ & \\
\hline 2455252.72577 & $148.9 \pm 10.2$ & \\
\hline 2455252.72940 & $146.5 \pm 10.0$ & \\
\hline 2455252.73303 & $143.8 \pm 10.0$ & \\
\hline 2455252.73666 & $135.4 \pm 10.0$ & \\
\hline 2455252.74029 & $126.9 \pm 10.1$ & \\
\hline 2455252.74392 & $115.1 \pm 10.0$ & \\
\hline 2455252.74755 & $104.0 \pm 10.0$ & \\
\hline 2455252.75118 & $91.9 \pm 9.9$ & \\
\hline 2455252.75481 & $76.7 \pm 9.9$ & \\
\hline
\end{tabular}

(e.g. Wood \& Saffer 1999; Drechsel et al. 2001), all spectra were fitted separately. This effect is linked to the reflection effect since the contribution of the companion to the spectra is stronger the more the heated side is visible.

The variation in the parameters over the phase is likewise clearly visible for our star system. The temperature seems to change about $1500 \mathrm{~K}$, and the surface gravity about 0.09 dex over the phase as can be seen in Fig. 3. The helium abundance shows a scatter of about $0.5 \mathrm{dex}$, which is not much more than the statistical error. For a grazing eclipse the least
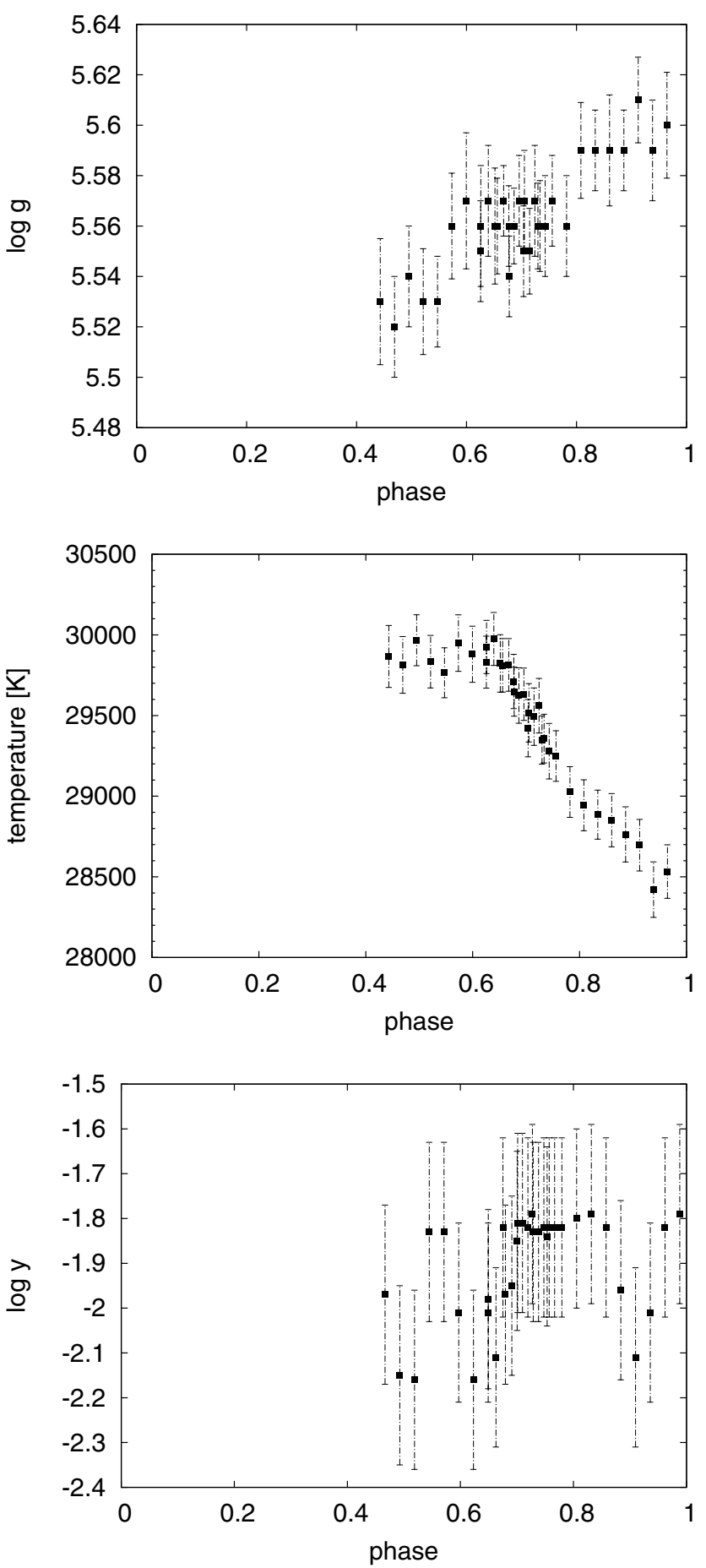

Fig. 3. Apparent variation of the atmospheric parameters over the phase. Temperature, surface gravity and helium abundance were measured from the Gemini/GMOS spectra. The errors are statistical errors.

contamination by the secondary, hence the most accurate, value is expected for phase 0 . Unfortunately, the binary was not observed at this phase. We adopted the value closest to phase zero and therefore a temperature of $28400 \pm 500 \mathrm{~K}$, a surface gravity of $\log g=5.60 \pm 0.05$ and a helium abundance of about $1.8 \pm 0.2$. We considered bigger errors than the statistical errors, which can be seen in Fig. 3, to account for the change in the parameters over the phase and systematic errors. The helium abundance is subsolar as in most sdBs. Such atmospheric parameters are typical of sdBs in HW Vir systems (see Fig. 4). 


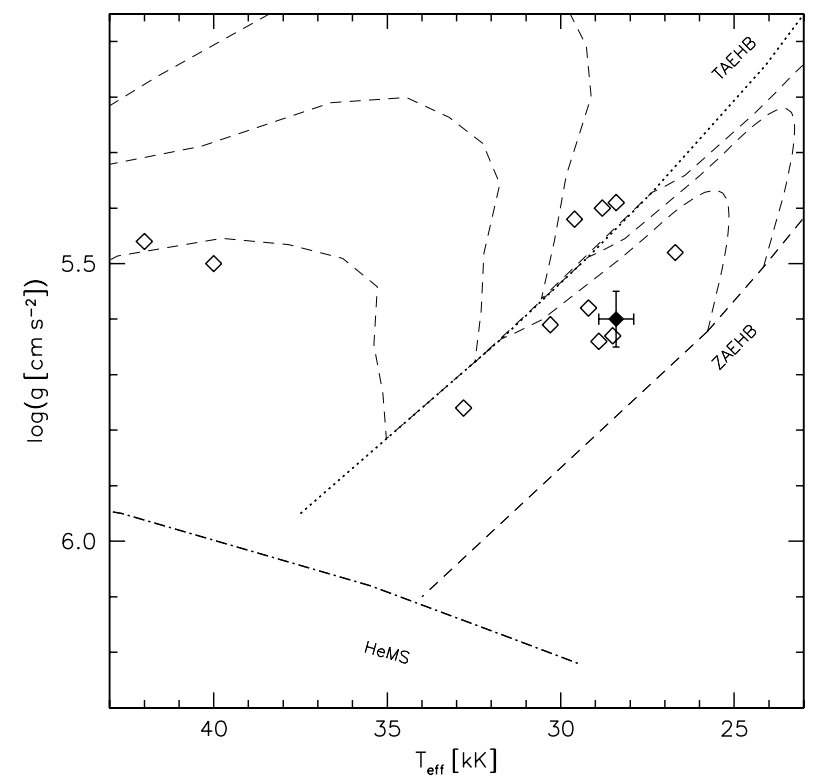

Fig. 4. $T_{\text {eff }}-\log g$-diagram. The helium main sequence (HeMS) and the EHB band (limited by the zero-age EHB, ZAEHB, and the terminal-age EHB, TAEHB) are superimposed with EHB evolutionary tracks from Dorman et al. (1993). The position of ASAS 10232 is indicated with a solid diamond. Open diamonds mark the position of other HW Vir-like systems (Charpinet et al. 2008; Drechsel et al. 2001; For et al. 2010; Geier et al. 2011b; Maxted et al. 2002; Klepp \& Rauch 2011; Østensen et al. 2008, 2010; Wood \& Saffer 1999; Almeida et al. 2012; Barlow et al. 2013).

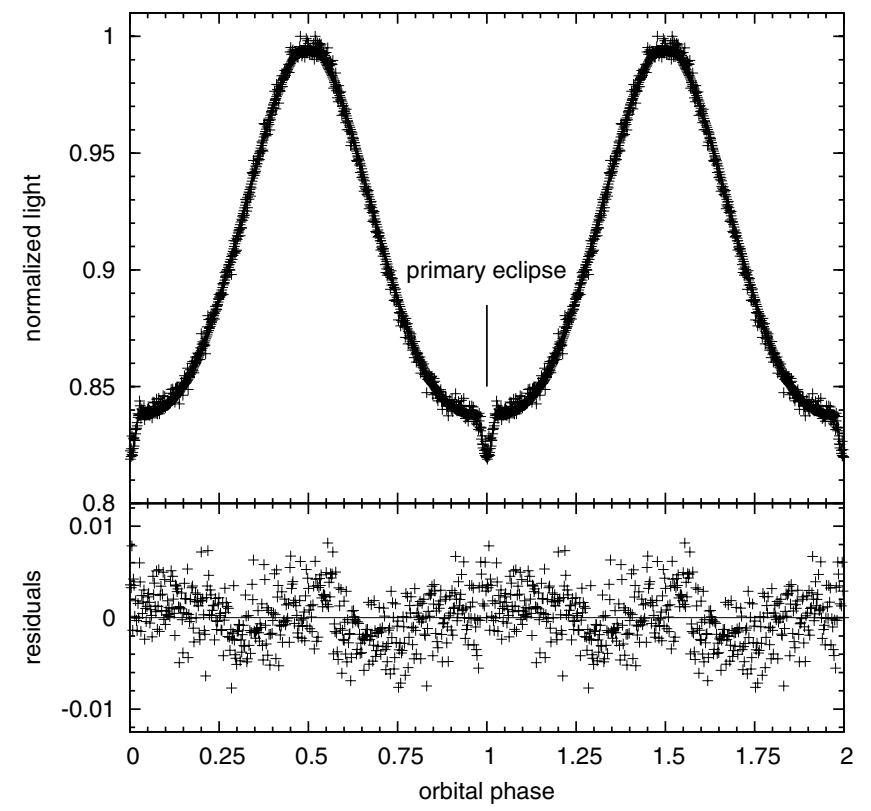

Fig. 5. Phased SuperWASP lightcurve. The solid line demonstrates the best-fitting model. In the bottom panel the residuals can be seen. The wave pattern that is seen in the residuals can be explained by the simplified treatment of the reflection effect. Better models of the reflection effect should remove this wave pattern.

\section{Photometric analysis}

\subsection{Ephemeris}

The SuperWASP lightcurve (see Fig. 5) clearly shows that ASAS 10232 is a short-period binary with a grazing eclipse. The

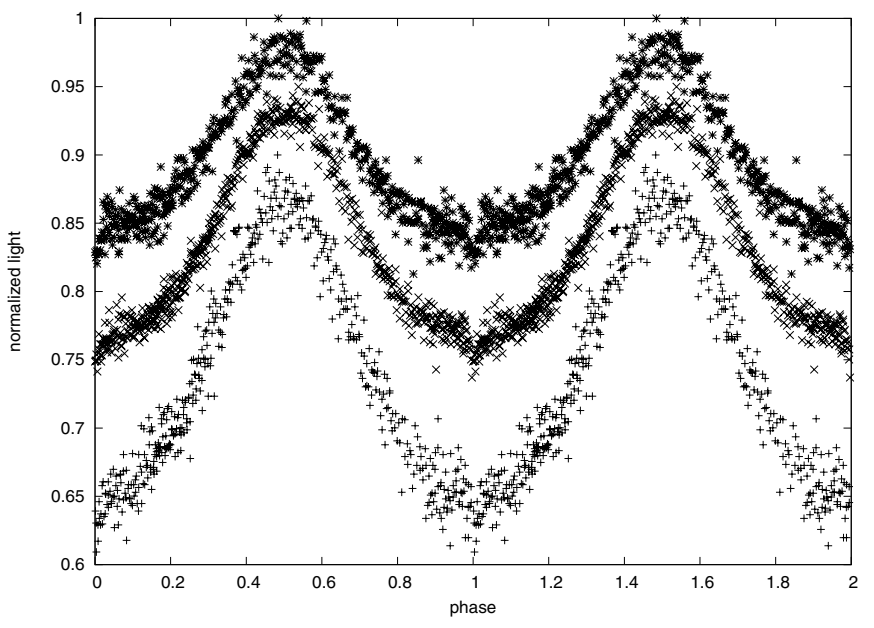

Fig. 6. Phased lightcurves of ASAS 10232 in the $B(*), V(\times)$, and $I(+)$ band taken from the Carnes Hill Observatory: $V$ is shifted by $0.05, I$ is shifted by 0.1 .

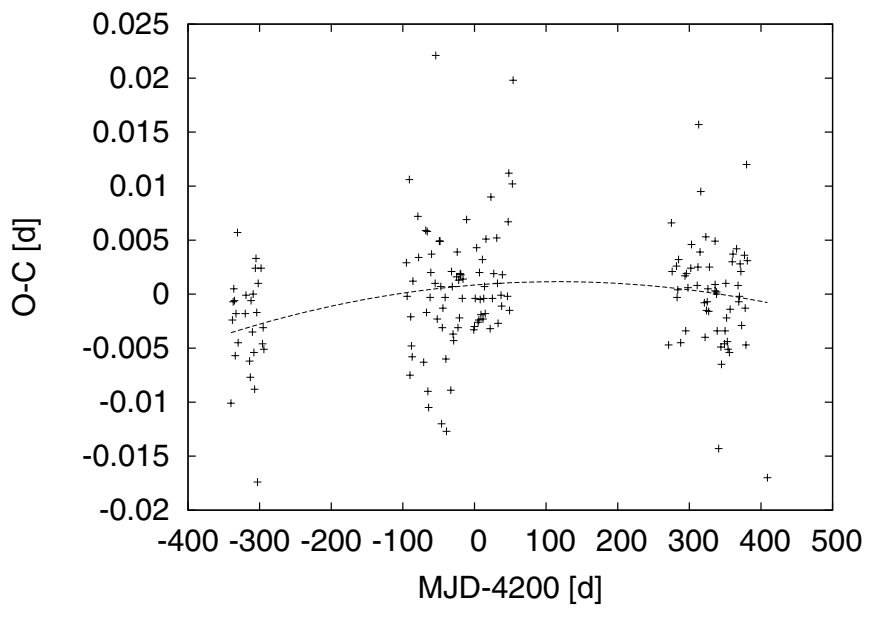

Fig. 7. O-C diagram of ASAS 10232 from the SuperWasp and ASAS observations with a fit of a parabola to determine a period derivative.

huge reflection effect indicates a cool main sequence star companion. The Carnes Hill Observatory lightcurves (see Fig. 6) in the three bands $B, V$, and $I$ were used to determine the time of the primary minimum. Parabolas were fitted to the minimum obtained on the night of 13 March 2008, where the minimum was clearly visible in all bands. From the different measured times of the minima in the different bands, the standard deviation was calculated as the error in time. Since the SuperWASP lightcurve has much higher accuracy and covers a longer time span it was used to determine the orbital period of the system. The period derivative was found by a fit of a parabola to the $\mathrm{O}-\mathrm{C}$ curve measured from the SuperWASP and BVI lightcurves, see Fig. 7:

$$
\begin{gathered}
\mathrm{HJD}=2454538.99689+0.13926940 \times E-6^{\mathrm{d}} .1 \times 10^{-11} \times E^{2} \\
\pm 42 \\
\pm 4 \quad \pm 2.3
\end{gathered}
$$

The period derivative is not yet a three-sigma detection, more observations are needed to confirm it.

\subsection{Lightcurve solution}

The phased $B, V, I$ lightcurves along with the white-light SuperWASP lightcurve were analysed with the MORO code 
Table 2. Parameters of ASAS 10232 ( TYC 7709-376-1).

\begin{tabular}{l|c|c|c}
\hline \hline Coordinates & \multicolumn{4}{|c}{$102322-373700(\mathrm{~J} 2000.0)$} \\
Proper motions & \multicolumn{2}{|c}{$-28.9-22.8[2.42 .435] \mathrm{mas} / \mathrm{yr}$} \\
\hline Mass of the sdB & $M_{\mathrm{sdB}}$ & {$\left[M_{\odot}\right]$} & $0.461 \pm 0.051$ \\
Mass of the companion & $M_{\mathrm{comp}}$ & {$\left[M_{\odot}\right]$} & $0.157 \pm 0.017$ \\
Separation & $a$ & {$\left[R_{\odot}\right]$} & $0.963 \pm 0.036$ \\
Mean radius of the sdB & $R_{\mathrm{sdB}}$ & {$\left[R_{\odot}\right]$} & $0.179 \pm 0.011$ \\
Mean radius of the comp & $R_{\mathrm{comp}}$ & {$\left[R_{\odot}\right]$} & $0.256 \pm 0.015$ \\
Surface gravity (phot) & $\log g(\mathrm{sdB})$ & & $5.60 \pm 0.02$ \\
Surface gravity (spec) & $\log g(\mathrm{sdB})$ & & $5.60 \pm 0.05$ \\
\hline
\end{tabular}

(Drechsel et al. 1995). This lightcurve solution program is based on the Wilson-Devinney approach (Wilson \& Devinney 1971), but uses a modified Roche model that considers the radiative pressure of hot binaries. For the analysis of the SuperWASP lightcurve normal points were formed by binning the fluxes of individual measurements over narrow time intervals. This is necessary because of the huge number of data points (13816 data points were binned to 478 normal points) that would make the analysis very tedious.

We used the Wilson-Devinney mode 2, which does not restrict the system configuration and links the luminosity and the temperature of the second component on the basis of the Planck law. Since the luminosity ratio is very high and the companion contributes almost exclusively via the reflection effect, the measured temperature of the companion is not reliable. Because of the high number of parameters $(12+5 \mathrm{~N}=32)$, some have to be deducted from spectral or theoretical constraints.

Owing the early spectral type of the primary star the gravity darkening exponent can be fixed at $g_{1}=1$ as expected for radiative outer envelopes (von Zeipel 1924). For the cool convective companion, $g_{2}$ was set to 0.32 (Lucy 1967). The linear limb darkening coefficients were interpolated from the tables of Wade $\&$ Rucinski (1985) and fixed at $x_{1}(B)=0.26, x_{1}(V)=0.22$, $x_{1}(I)=0.165, x_{1}($ white $)=0.20$. Since only monochromatic lightcurves are calculated in the lightcurve solution program, we used the central wavelengths of the band filters. The secondary's limb darkening coefficient $x_{2}$ was varied as the limb darkening of such heated objects cannot be predicted. The temperature of the hot component was taken from the spectral analysis $\left(T_{\text {eff }, \text { sdB }}=28400 \mathrm{~K}\right)$.

For the albedos of the companion values exceeding 1 were needed to model the reflection effect. This can be explained with processes in the stellar atmosphere that cause a spectral redistribution of the irradiated energy with wavelength. The third light that accounts for the disturbance by a potential third object in the system was first varied but then set to 0 because it did not deviate from this value. As the spectral type of the companion is very late, radiation pressure does not play a role, and the radiation pressure coefficient for the companion was fixed to zero (cf. Drechsel et al. 1995, 2001).

The rest of the parameters, such as the radiation pressure coefficient for the primary star, the inclination, the effective temperature of the companion, and the Roche potentials, were adjusted. A grid with different fixed mass ratios and different start parameters was calculated. All four lightcurves were analysed simultaneously. That there are so many correlated parameters means a unique solution cannot be found from the lightcurve alone, because of the degeneracy of mass ratio and radii of the components. In Table 3 the parameters of the lightcurve solution with the best standard deviation can be found.
Table 3. Best lightcurve solution of ASAS 10232.

\begin{tabular}{|c|c|c|}
\hline \multicolumn{3}{|c|}{ Fixed parameters: } \\
\hline$q\left(=M_{2} / M_{1}\right)$ & & 0.34 \\
\hline$T_{\text {eff }}(1)$ & {$[\mathrm{K}]$} & 28400 \\
\hline$g_{1}^{b}$ & & 1.0 \\
\hline$g_{2}^{b}$ & & 0.32 \\
\hline$x_{1}(B)^{c}$ & & 0.26 \\
\hline$x_{1}(V)^{c}$ & & 0.22 \\
\hline$x_{1}(I)^{c}$ & & 0.165 \\
\hline$x_{1}(\text { white })^{c}$ & & 0.20 \\
\hline$\delta_{2}^{d}$ & & 0.0 \\
\hline$l_{3}^{f}$ & & 0.0 \\
\hline \multicolumn{3}{|c|}{ Adjusted parameters: } \\
\hline$i$ & {$\left[{ }^{\circ}\right]$} & $65.86 \pm 0.69$ \\
\hline$T_{\text {eff }}(2)$ & {$[\mathrm{K}]$} & $3380 \pm 561$ \\
\hline$A_{1}{ }^{a}$ & & $0.94 \pm 0.03$ \\
\hline$A_{2}{ }^{a}$ & & $1.21 \pm 0.13$ \\
\hline$\Omega_{1}^{f}$ & & $5.700 \pm 0.260$ \\
\hline$\Omega_{2}^{f}$ & & $2.673 \pm 0.052$ \\
\hline$\frac{L_{1}}{L_{1}+L_{2}}(B)^{g}$ & & $0.99954 \pm 0.00077$ \\
\hline$\frac{L_{1}}{L_{1}+L_{2}}(V)^{g}$ & & $0.99804 \pm 0.00247$ \\
\hline$\frac{L_{1}}{L_{1}+L_{2}}(I)^{g}$ & & $0.99036 \pm 0.00845$ \\
\hline$\frac{L_{1}+L_{1}}{L_{1}+L_{2}}(\text { white })^{g}$ & & $0.99709 \pm 0.00337$ \\
\hline$\delta_{1}$ & & $0.0123 \pm 0.0080$ \\
\hline$x_{2}(B)$ & & $0.638 \pm 0.097$ \\
\hline$x_{2}(V)$ & & $0.548 \pm 0.0630$ \\
\hline$x_{2}(I)$ & & $0.625 \pm 0.087$ \\
\hline$x_{2}$ (white) & & $0.238 \pm 0.089$ \\
\hline \multicolumn{3}{|l|}{${\text { Roche } \text { radii }^{h} \text { : }}$} \\
\hline$r_{1}($ pole $)$ & [a] & $0.184 \pm 0.009$ \\
\hline$r_{1}$ (point) & [a] & $0.186 \pm 0.00$ \\
\hline$r_{1}($ side $)$ & [a] & $0.185 \pm 0.009$ \\
\hline$r_{1}$ (back) & [a] & $0.186 \pm 0.009$ \\
\hline$r_{2}($ pole $)$ & [a] & $0.248 \pm 0.009$ \\
\hline$r_{2}$ (point) & [a] & $0.286 \pm 0.017$ \\
\hline$r_{2}($ side $)$ & [a] & $0.256 \pm 0.010$ \\
\hline$r_{2}$ (back) & [a] & $0.277 \pm 0.014$ \\
\hline
\end{tabular}

Notes. ${ }^{(a)}$ Bolometric albedo. ${ }^{(b)}$ Gravitational darkening exponent. (c) Linear limb darkening coefficient; taken from Wade \& Rucinski (1985). ${ }^{(d)}$ Radiation pressure parameter, see Drechsel et al. (1995). ${ }^{(e)}$ Fraction of third light at maximum. ${ }^{(f)}$ Roche potentials. ${ }^{(g)}$ Relative luminosity; $L_{2}$ is not independently adjusted, but recomputed from $r_{2}$ and $T_{\text {eff }}(2){ }^{\left({ }^{h}\right)}$ Fractional Roche radii in units of separation of mass centers.

The Euler lightcurves are consistent with this solution as can be seen in Fig. 8. The only difference is a higher albedo of 1.3 for the companion. Because the Euler lightcurves have a good signal-to-noise and four different colour bands are covered, the higher albedo is probably preferable.

Figure 9 shows the equipotential surfaces of both components for the case of the best solutions over the phase. It is clearly visible that ASAS 10232 is a detached system with a grazing eclipse since both components stay inside their Roche lobe. The companion is bigger than the primary star and is slightly distorted.

\section{System parameters}

Since the radial velocity amplitude of the companion is not known and a degeneracy in the mass ratio appears in the 


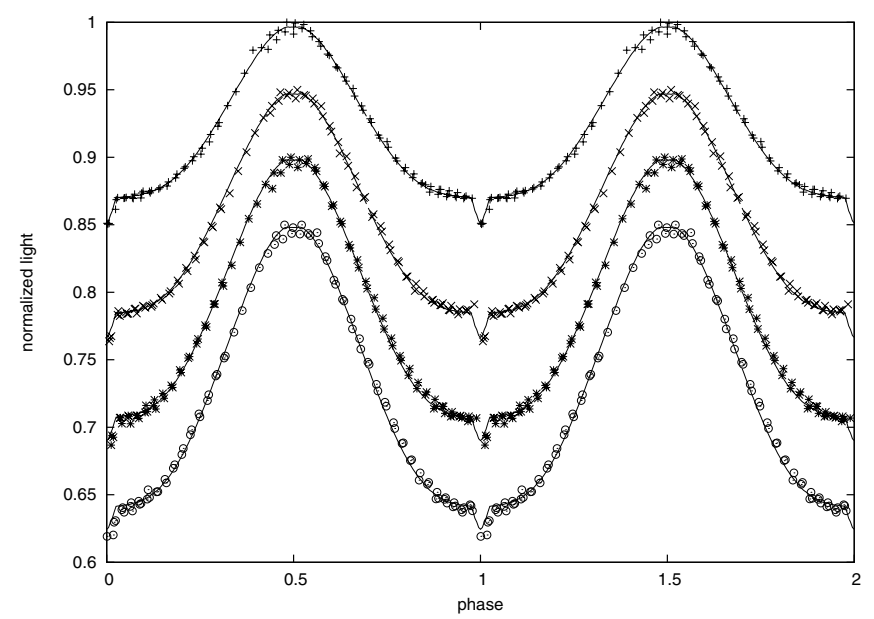

Fig. 8. BVRI Euler lightcurves with the model from the parameters of the best solution, but a higher $A_{2}=1.3$, every lightcurve is shifted by 0.05 .
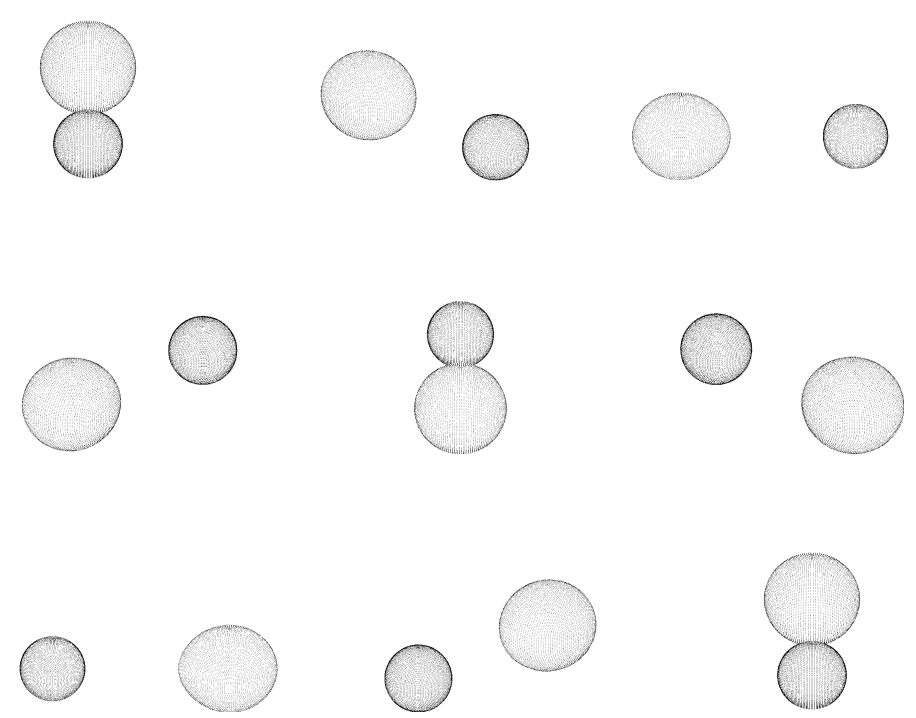

Fig. 9. Equipotential structure of ASAS 10232 corresponding to the best fit solution over the phase from 0.5 to 0.5 in steps of 0.125 .

lightcurve solutions, we do not have a unique solution of the system yet. The solution with the smallest standard deviation from the lightcurve model to the measured values is found for a mass ratio $q=0.34$. For this mass ratio we derive an inclination angle of $i=65.9^{\circ}$ from the lightcurve analysis. Furthermore, the mean radii for the components could be determined: $R_{1} / a=0.185$ and $R_{2} / a=0.266$, where $a$ is the separation of the mass centres. Together with the $K_{1}=81.0 \pm 3.0 \mathrm{~km} \mathrm{~s}^{-1}$ measured from the radial velocity curve and the mass ratio $q$, the inclination $i$ and the period $P=0.13927 \mathrm{~d}$, determined from the lightcurve, the masses of the components $M_{1}=0.461 \pm 0.051 M_{\odot}$ and $M_{2}=$ $0.157 \pm 0.017 M_{\odot}$ were derived. Moreover, the separation $a=$ $0.963 \pm 0.036 R_{\odot}$, hence the absolute values for the radii of the stars $R_{\mathrm{sdB}}=0.179 \pm 0.011 R_{\odot}$ and $R_{\text {comp }}=0.256 \pm 0.015 R_{\odot}$, could be calculated.

Radius and mass of the companion leads to the conclusion that the companion must be an M dwarf. The derived mass for the sdB is consistent with theoretical calculations for the formation of sdBs via the common envelope channel (Han et al. 2002, 2003).
To check this solution the surface gravity determined from the spectra can be compared to a photometric surface gravity. This surface gravity is derived via the mass-radius relation from the mass and radius calculated from the radial velocity curve together with the lightcurve analysis. The surface gravity determined in this way $\log g$ (phot) $=5.60 \pm 0.02$ is in perfect agreement to the spectroscopic surface gravity $\log g(\mathrm{spec})=$ $5.60 \pm 0.05$.

\section{Conclusions}

We discovered the new eclipsing sdB star ASAS 102322-3737.0, which shows a grazing eclipse and a huge reflection effect that is the only contribution of the companion to the optical light of the system. It has an orbital period of $P=0.13927 \mathrm{~d}$ and an inclination of $i=65.9^{\circ}$. A unique solution cannot be found because of a degeneracy in the mass ratio. The best lightcurve solution was calculated for a mass ratio of $q=0.34$. The masses of the components for this solution $M_{\mathrm{sdB}}=0.461 \pm 0.051 M_{\odot}$ and $M_{\text {comp }}=0.157 \pm 0.017 M_{\odot}$ are typical of an sdB and a late main sequence star. Also the spectroscopic and the photometric surface gravity are in agreement. Since ASAS 10232 is very bright it is possible to apply rapid high-resolution time-series spectroscopy to search for spectral features and the RV semiamplitude from the companion to resolve the degeneracy in the mass ratio, as done for AA Dor (Vučković et al. 2008).

Some of the sdBs were found to be pulsating. There are two classes of sdB pulsators, p-mode pulsators with periods of some minutes and g-mode pulsators with periods of 0.5 to $2 \mathrm{~h}$, which are separated by their atmospheric parameters. Until now only one pulsating HW Virginis system was found, NY Virginis (Vučković et al. 2007). Such systems are very interesting since it is possible to compare the results from asteroseismology to the results from the lightcurve analysis of the eclipses. If it is pulsating, the parameters of ASAS 10232 would suggest that it is most likely a g-mode pulsator. However, the Euler lightcurve with the best signal-to-noise shows no sign of pulsation. But the strong reflection effect could hide the pulsations.

Period variations have been found for almost all of the HW Vir systems that have accurate eclipse timings covering more than five years, which may be attributed to the presence of a third body. In several cases the third bodies are likely to be one (or two) giant planet(s). These discoveries were unexpected, because it is considered to be difficult for giant planets to form around main sequence binaries owing to the short lifetime of circumbinary disks. In addition, such planets may not be able to survive common envelope (CE) evolution. Instead, it has been suggested that these circumbinary planets are second generation (Zorotovic \& Schreiber 2013), which is formed from the instability of a post-CE disk. Zorotovic \& Schreiber (2013) also proposed an alternative scenario for the period variations due to processes acting in deeply convective secondary stars.

HW Vir has been monitored for more than 28 years now and, Lee et al. (2009) found two sinusoidal variations of the lighttravel time for HW Vir from 24 years of data, suggesting there are two substellar objects orbiting the close binary. New observations by Beuermann et al. (2012), however, deviate significantly from the prediction of Lee et al. (2009). The new solution involves one planet and a brown dwarf or low-mass star orbiting the HW Vir binary (Beuermann et al. 2012). The new solution was found to be stable in contrast to that of Lee et al. (2009).

These findings suggest that the probablity of finding period variations in ASAS 10232 as well is high, whether due to substellar objects or the active secondary. The lesson learned 
from HW Vir is that long-term and dense monitoring is a prerequisite (Beuermann et al. 2012). ASAS is the third brightest HW Vir system known: only one magnitude fainter than HW Vir, which allows us to use readily accessible small telescopes to obtain lightcurves. It is therefore a promising target for amateur and secondary school observatories to team up with professional astronomers (Backhaus et al. 2012).

Acknowledgements. Based on observations at the La Silla Observatory of the European Southern Observatory for programme number 080.D-0685(A) and on observations obtained at the Gemini Observatory (Program ID: GS-2009BQ-98), which is operated by the Association of Universities for Research in Astronomy, Inc., under a cooperative agreement with the NSF on behalf of the Gemini partnership: the National Science Foundation (United States), the Science and Technology Facilities Council (United Kingdom), the National Research Council (Canada), CONICYT (Chile), the Australian Research Council (Australia), Ministério da Ciência e Tecnologia (Brazil) and Ministerio de Ciencia, Tecnología e Innovación Productiva (Argentina). S.G. is supported by the Deutsche Forschungsgemeinschaft (DFG) through grant HE1356/49-1. R.Ø. acknowledges funding from the European Research Council under the European Community's Seventh Framework Programme (FP7/2007-2013)/ERC grant agreement №227224 (PROSPERITY), as well as from the Research Council of K.U.Leuven grant agreement GOA/2008/04. V.S. acknowledges funding by the Deutsches Zentrum für Luft- und Raumfahrt (grant 50 OR 1110) and by the Erika-Giehrl-Stiftung.

\section{References}

Almeida, L. A., Jablonski, F., Tello, J., \& Rodrigues, C. V. 2012, MNRAS, 423, 478

Backhaus, U., Bauer, S., Beuermann, K., et al. 2012, A\&A, 538, A84

Barlow, B. N., Kilkenny, D., Drechsel, H., et al. 2013, MNRAS, 430, 22

Beuermann, K., Dreizler, S., Hessman, F. V., \& Deller, J. 2012, A\&A, 543, A138

Brown, T. M., Ferguson, H. C., Davidsen, A. F., \& Dorman, B. 1997, APJ, 482, 685

Brown, T. M., Bowers, C. W., Kimble, R. A., Sweigart, A. V., \& Ferguson, H. C. 2000, APJ, 532, 308

Charpinet, S., van Grootel, V., Reese, D., et al. 2008, A\&A, 489, 377
Dorman, B., Rood, R. T., \& O’Connell, R. W. 1993, APJ, 419, 596 Drechsel, H., Haas, S., Lorenz, R., \& Gayler, S. 1995, A\&A, 294, 723 Drechsel, H., Heber, U., Napiwotzki, R., et al. 2001, A\&A, 379, 893

For, B.-Q., Green, E. M., Fontaine, G., et al. 2010, APJ, 708, 253

Geier, S., Maxted, P. F. L., Napiwotzki, R., et al. 2011a, A\&A, 526, A39

Geier, S., Schaffenroth, V., Drechsel, H., et al. 2011b, APJ, 731, L22

Geier, S., Marsh, T. R., Dunlap, B. H., et al. 2012 [arXiv: 1209.4740]

Han, Z., Podsiadlowski, P., Maxted, P. F. L., Marsh, T. R., \& Ivanova, N. 2002, MNRAS, 336, 449

Han, Z., Podsiadlowski, P., Maxted, P. F. L., \& Marsh, T. R. 2003, MNRAS, 341, 669

Heber, U. 1986, A\&A, 155, 33

Heber, U., Reid, I. N., \& Werner, K. 2000, A\&A, 363, 198

Hirsch, H. 2009, Ph.D. Thesis, Friedrich Alexander Universität Erlangen Nürnberg

Klepp, S., \& Rauch, T. 2011, A\&A, 531, L7

Lee, J. W., Kim, S.-L., Kim, C.-H., et al. 2009, AJ, 137, 3181

Lucy, L. B. 1967, Z. Astrophysik, 65, 89

Maxted, P. f. L., Heber, U., Marsh, T. R., \& North, R. C. 2001, MNRAS, 326, 1391

Maxted, P. F. L., Marsh, T. R., Heber, U., et al. 2002, MNRAS, 333, 231

Napiwotzki, R., Karl, C. A., Lisker, T., et al. 2004, Ap\&SS, 291, 321

Østensen, R. H., Oreiro, R., Hu, H., Drechsel, H., \& Heber, U. 2008, in Hot Subdwarf Stars and Related Objects, eds. U. Heber, C. S. Jeffery, \& R. Napiwotzki, ASP Conf. Ser., 392, 221

Østensen, R. H., Green, E. M., Bloemen, S., et al. 2010, MNRAS, 408, L51

Pojmanski, G. 2003, Acta Astron., 53, 341

Pollacco, D. L., Skillen, I., Cameron, A. C., et al. 2006, PASP, 118, 1407

Qian, S.-B., Zhu, L.-Y., Zola, S., et al. 2009, APJ, 695, L163

Saffer, R. A., Bergeron, P., Koester, D., \& Liebert, J. 1994, APJ, 432, 351

Vennes, S., Kawka, A., O’Toole, S. J., Németh, P., \& Burton, D. 2012, ApJ, 759, L25

von Zeipel, H. 1924, MNRAS, 84, 665

Vučković, M., Aerts, C., Östensen, R., et al. 2007, A\&A, 471, 605

Vučković, M., Østensen, R., Bloemen, S., Decoster, I., \& Aerts, C. 2008, in Hot Subdwarf Stars and Related Objects, eds. U. Heber, C. S. Jeffery, \& R. Napiwotzki, ASP Conf. Ser., 392, 199

Wade, R. A., \& Rucinski, S. M. 1985, A\&AS, 60, 471

Wilson, R. E., \& Devinney, E. J. 1971, APJ, 166, 605

Wood, J. H., \& Saffer, R. 1999, MNRAS, 305, 820

Zorotovic, M., \& Schreiber, M. R. 2013, A\&A, 549, A95 